DOI: $10.217672573-5365.100021$

\title{
Spinal Cord Injury Induced Hyperalgesia is Associated with Increased Levels of Let-7 Family of MicroRNAs
}

\author{
Carlene Callahan ${ }^{1}$, Michelle Yanik ${ }^{1}$, Dorothy Dobbins ${ }^{2}$ and Kori L Brewer ${ }^{1 *}$ \\ ${ }^{1}$ Department of Emergency Medicine, East Carolina University Brody School of Medicine, Greenville NC 27834, USA \\ ${ }^{2}$ Department of Neuroscience, Wake Forest University, Medical Center Blvd, Winston-Salem NC 27157, USA
}

*Corresponding author: Kori L Brewer, East Carolina University, Brody School of Medicine, Dept. of Emergency Medicine, 600 Moye Blvd, MailStop \#625, Greenville NC 27834, USA, Tel: 252-744-2159; Fax: 252-744-3589; E-mail: brewerk@ecu.edu

Rec date: Feb 19, 2020Acc date: march 13, 202Bub date: April 26, 2020

Citation: Callahan C, Yanik M, Dobbins D, et al. Spinal cord injury induced hyperalgesia is associated with increased levels of Let-7 family of MicroRNAs. Cell Mol Med. 2020, 6:1.

\section{Abstract}

Title: Spinal cord injury induced hyperalgesia is associated with increased levels of Let-7 family of MicroRNAs.

Background: Spinal cord injury $(\mathrm{SCl})$ results in chronic pain syndromes that are often refractory to treatment with opioids, and may represent mu opioid receptor (MOR) dysfunction. MicroRNAs play a role in gene regulation by binding to target mRNA, causing translational repression or mRNA degradation. Let-7 family microRNAs have been shown to target MOR suggesting they may influence the development of pain states and opioid responsiveness. This study sought to determine temporal changes in Let-7 family miRNA and correlate them to the onset of pain behaviors in a rodent model of post-SCI pain.

Methods and findings: Thermal thresholds (evoked pain) were assessed in rats before and after intramedullary injection of quisqualic acid $(n=18)$ or sham laminectomy $(n=18)$. Three of the Let-7 family miRNA levels were determined (Let-7a, Lets-7c and Let 7g) using RT-PCR and qRT-PCR on spinal cord segments caudal to lesion at 7,14 and 21 days after injury. Thermal thresholds were significantly reduced compared to baseline in injured animals at day 21 (mean decrease of $1.9 \mathrm{sec} ; \mathrm{p}=0.02 ; 95 \%$ $\mathrm{Cl}=0.41-3.3$ ). Sham animals showed no significant change in thermal thresholds over time $(\mathrm{p}=0.77 ; 95 \% \mathrm{Cl}=3.2-4.2)$. $\mathrm{SCl}$ led to a trend in increasing Let-7 family miRNA levels over time. Compared to sham animals, miRNA levels studied increased in injured animals at 14 and 21 days, corresponding to the period during which post-injury pain developed.

Conclusions: Our data suggests that increased levels of Let-7 family miRNAs are involved in behavioral responses to $\mathrm{SCl}$. Future work will examine if inhibition of Let-7 family miRNAs alters the behavioral and cellular response to $\mathrm{SCl}$.
Keywords: Spinal cord injury; Hyperalgesia; Mu opioid receptor; Let-7 family miRNA; Neuropathic pain; Quisqualate

Abbreviations: SCl: Spinal Cord Injury; miRNA: microRNA; MOR: Mu Opioid Receptor; NIH: National Institutes of Health; QUIS: Quisqualic Acid; PCR: Polymerase Chain Reaction

\section{Introduction}

Chronic pain syndromes following spinal cord injury (SCl) are common and are a major challenge facing injured individuals due to the lack of universally effective treatment options [1-4]. Chronic pain after injury strongly reduces quality of life, affecting both the physical and mental health of patients, and can interfere with rehabilitative efforts [5-9]. Opioids are commonly employed for the treatment of $\mathrm{SCl}$-related pain do not provide meaningful analgesia in all $\mathrm{SCl}$ patients $[10,11]$. Functional changes to the mu opioid receptor (MOR) have been observed following $\mathrm{SCl}$, providing a potential explanation for why pain mitigation with opioids may be unsuccessful [12]. However, the mechanism behind these changes is not well understood

MicroRNAs, small noncoding RNAs, are an emerging player in the field of pain research and have the potential to elucidate the chronic pain sequela associated with $\mathrm{SCl}$ at cellular level [13-19]. The Let-7 family of miRNAs has specifically been shown to target MOR, suggesting its link to opioid tolerance [20]. In addition, this family of miRNA shows increased levels following $\mathrm{SCl}$ [21]. Together, these studies suggest that the Let-7 family of miRNA may play a role in the development of pain as well as the reduction of effective analgesia with opioids after $\mathrm{SCl}$.

The current study sought to determine a time line of the post-translational modification of opioid receptors, by measuring the quantitative changes of the Let-7 family miRNA (Let-7a, Let-7c, and Let-7g) after experimental $\mathrm{SCl}$ in rats, and correlate those changes to the onset of hyperalgesia, a measure of below-level $\mathrm{SCl}$ pain clinically. 


\section{Methods}

\section{Animal group}

Adult male Long Evans rats (250-300g) were randomized to receive either excitotoxic $\mathrm{SCl}(\mathrm{n}=20)$ or SHAM laminectomy as control $(n=20)$. Ten animals from each group were used for behavioral experiments described below, and the remaining 10 animals from each group used for molecular studies $(n=5$ each for 7 and 14 days time points). All experimental protocols were reviewed and approved by the institutional animal care and use committee of east Carolina university and all animal procedures followed the national institutes of health $(\mathrm{NIH})$ guidelines for the care and use of laboratory animals.

\section{Surgical procedures}

Rodents were anesthetized with inhaled isofluorane ( $2 \%$ to $3 \%$ to effect) and placed in ear bars of a stereotactic apparatus. The back of the animal was shaved, scrubbed with betadine and a $3 \mathrm{~cm}$ to $4 \mathrm{~cm}$ midline incision made. The vertebral column was exposed and a laminectomy performed to remove both the spinous process and lamina of a single segment between spinal levels T12-L1. The vertebral column was then stabilized using a spinal clamp. For $\mathrm{SCl}$ animals, a total volume of $1.2 \mu \mathrm{l}$ of quisqualic acid (QUIS) was injected unilaterally (right side) into the deep dorsal gray matter 1.0 $\mathrm{mm}$ below the dorsal surface of the cord using glass micropipettes attached to a Hamilton syringe and mounted on a microinjector. Following injections, the muscles were sutured together and the skin was closed using staples. Control animals received laminectomy without injection and were otherwise treated identically to the experimental group.

\section{Behavioral testing}

Assessment of evoked pain was performed on 10 QUIS and 10 SHAM animals using the Hargreaves test [22], which measures the latency to withdraw a paw from a $52^{\circ} \mathrm{C}$ heat stimulus. Rats were acclimated to the testing room and apparatus for 1 hour/day for 3 days prior to testing. On test days ( 2 days prior to surgery and again on 7, 14 and 21 days after surgery), the right hind paws of animals were tested 5 times in total with 10 minutes in-between each trial to prevent sensitization of the skin. High and low values were discarded and the remaining 3 values averaged to provide a mean latency $( \pm S E M)$ to withdrawal for each day. After the last behavioral test was performed, tissue was harvested for confirmation of lesion or PCR experiments as described below.

\section{Confirmation of dorsal horn injury}

At the conclusion of behavioral testing, animals were deeply anesthetized with inhaled isoflurane and transcardially perfused with $500 \mathrm{ml}$ of cold saline followed by $500 \mathrm{ml}$ of $4 \%$ paraformaldehyde. Segments of spinal cords corresponding to the level of injury were removed and placed in $4 \%$ paraformaldehyde overnight at $4{ }^{\circ} \mathrm{C}$ and then switched to $30 \%$ sucrose for at least 48 hours. A sliding microtome with a freezing stage was used to cut $75 \mu \mathrm{m}$ thick serial sections through the lesion site. Sections were mounted onto gelatincoated slides and stained with cresyl violet to visualize gross damage to the cord.

\section{Analysis of Let-7 family microRNA levels in spinal cords after injury}

Lumbar spinal cord segments were removed fresh from QUIS and SHAM animals that had survived for $7(n=5), 14(n=5)$ or $21(n=5)$ days after surgery. Tissue was immediately frozen in liquid nitrogen and stored at $-80^{\circ} \mathrm{C}$ until ready for use. Total microRNA was later extracted from the spinal cord tissue using mirVANA $^{\mathrm{TM}}$ miRNA Isolation Kit (Life Technologies) and total RNA quantified by Nanodrop ND-1000 (Nanodrop Technologies). Total RNA from each animal was transcribed into single-stranded cDNA using the reverse primer PolyT. RTPCR was performed in a $15 \mu$ l solution containing $1,000 \mathrm{ng}$ total RNA, DNase-/RNase-free water, $0.19 \mu$ l RNase inhibitor (20 U/ $\mu \mathrm{l}), 0.15 \mu \mathrm{l}$ 100mM dNTPs, 10x RT buffer, $1 \mu \mathrm{l}$ MultiScribe $^{\mathrm{TM}}$ reverse transcriptase $(50 \mathrm{U} / \mu \mathrm{l})$ and $2 \mu \mathrm{l}$ Poly(T) primer mix (See Table 1 for primer sequences). Reactions were performed using an Ependorf Mastercycler ${ }^{\circledR}$ Pro PCR machine. The cDNA was amplified using quantitative real time PCR (qRT$P C R$ ) in $15 \mu$ solutions containing $5.5 \mu \mathrm{l}$ DNase/RNase free water, $7.5 \mu$ SYBR green PCR master mix, $1 \mu \mathrm{l}$ RT-PCR product and $1 \mu \mathrm{l}$ primer mix. Reactions were performed using $\mathrm{Vii}^{\mathrm{TM}} 7$ real-time PCR system with $10 \mathrm{~min}$. polymerase activation $\left(95^{\circ} \mathrm{C}\right)$, denaturation for $15 \mathrm{sec}\left(95^{\circ} \mathrm{C}\right)$ and an annealing step for $60 \mathrm{sec}\left(60^{\circ} \mathrm{C}\right)$. Denaturation and annealing were repeated for 40 cycles with GAPDH used as a reference gene for normalizing QRT-PCR results (Forward Primer: TGCACCACCAACTGCTTAGC; Reverse Primer: GGCATGGACTGTGGTCATGAG). The $\triangle \triangle$ Ct method was used for analysis [23] and fold-change for each miRNA in QUIS animals reported as compared to SHAM animals (i.e. ratio of QUIS/ SHAM miRNA levels).

Table 1 Primer sequences

\begin{tabular}{|c|l|l|}
\hline miRnA & Forward & Reverse \\
\hline Let-7a & GCGgCGgTGAGGTAGTAGGTTG & GTCGTATCCAGTGCAGGGTCCGAGGTATTCGCACTGGATACGACAACTAT \\
\hline Let-7g & GCGGCGGTGAGGTAGTAGTTTG & GTCGTATCCAGTGCAGGGTCCGAGGTATTCGCACTGGATACGACAACTGT \\
\hline Let-7c & GCGGCGGTGAGGTAGTAGGTTG & GTCGTATCCAGTGCAGGGTCCGAGGTATTCGCACTGGATACGACAACCAT \\
\hline
\end{tabular}




\section{Statistical analysis}

For behavior data, withdrawal thresholds were compared within groups across time using a repeated measure analysis of variance (ANOVA) followed by post-hoc tests (LSD) when appropriate. Levels of each miRNA measured in QUIS vs. SHAM injected animals were compared at each survival time using two sample t-test. miRNA levels in QUIS injected animals were compared across survival times with one-way ANOVA to determine differences in fold changes between time points. In all cases, $p<0.05$ was used to indicate significance.

\section{Results}

\section{Histological outcomes}

All QUIS injected animals showed evidence of neuronal loss in the deep dorsal gray matter, primarily restricted to Lamina III-VI. Representative examples of damage seen in QUIS and SHAM animals are shown in Figure 1.

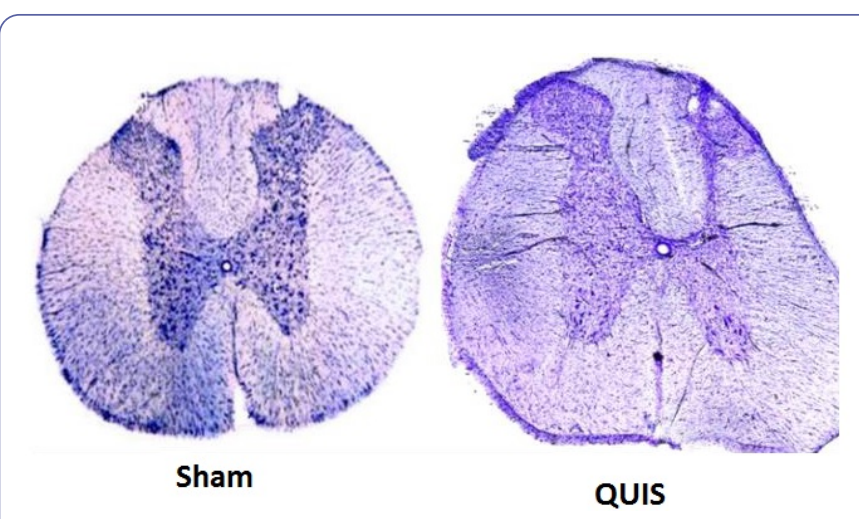

Figure 1 Representative pattern of neuronal loss caused by intramedullary injection of quisqualate. Cresyl violet staining shows normal morphology of spinal gray matter in sham animal. In QUIS-injected spinal cord, the gray matter below the superficial dorsal horn is significantly narrowed as a result of neuronal death.

\section{Behavioral outcomes}

Excitotoxic $\mathrm{SCl}$ led to significantly reduced thermal withdrawal latencies over time compared to baseline, indicative of hyperalgesia $(10.2 \pm 0.69 \mathrm{sec}$. at baseline vs. $8.3 \pm$ $0.38 \mathrm{sec}$. at 21 days; mean difference $=-1.86 ; \mathrm{p}=0.02 ; 95 \%$ $\mathrm{Cl}=0.41-3.3)$. In contrast, thresholds from SHAM animals remained constant over time $(10.8 \pm 1.12 \mathrm{sec}$. at baseline vs. $10.3 \pm 1.13 \mathrm{sec}$. at 21 days; mean difference $=0.49 ; p=0.77 ; 95 \%$ $\mathrm{Cl}=-3.2-4.2$; Figure 2).

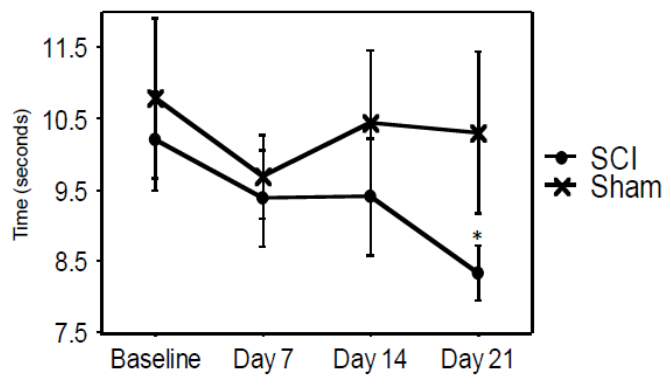

Figure 2 The effects of intramedullary injection of quisqualate or sham surgery on time to withdraw from a thermal stimulus delivered to the hind paw. Each point represents the mean \pm SEM of all trials $(n=3)$ for all animals on each day of testing. Thermal thresholds to heat remained stable over the 21 days survival period in sham animals with no significant change from baseline (mean difference $=0.5 \mathrm{sec} ; \mathrm{p}=0.76$ ). In contrast, withdrawal latencies from a thermal stimulus significantly declined from baseline to 21 days post-injury in QUIS-injected animals (mean difference $=1.9 \mathrm{sec} ; \mathrm{p}=0.02$ ).

Let-7 microRNA levels in QUIS vs. SHAM animals. MicroRNA from the Let-7 family was analyzed using PCR techniques on spinal cord tissue caudal to injury site. Let-7 a, c, and g were chosen as representatives of the Let- 7 microRNA family due to their highly conserved sequences. When normalized to GAPDH, the mean fold-change ( \pm SEM) in SHAM animals for the miRNAs studied were $0.23 \pm 0.1$ (Let-7a), $0.28 \pm 0.1$ (Let-7c) and $0.48 \pm 0.3$ (Let-7g). At 7 days post-surgery, Let-7c was significantly increased in $\mathrm{SCl}$ animals compared to sham animals $(p<0.05)$. At 14 and 21 days post-surgery, each of the miRNAs studied were significantly increased in $\mathrm{SCl}$ animals compared to SHAM animals. ( $p<0.05$; Figure 3 ) indicating that the changes seen in Let-7 levels were not a general response to surgery.

Let-7 microRNA levels in QUIS animals across time points. There were no significant differences between the 3 family members of Let- 7 studied at any time point ( $>00.05)$. Mean levels for all 3 miRNAs studied increased with time after injury with the lowest levels seen at 7 days after injury (Let $7 a=1.1 \pm$ 0.2 ; Let $7 \mathrm{c}=1.6 \pm 0.2$; Let $7 \mathrm{~g}=1.2 \pm 0.1$ ) and highest at 21 days after injury (Let $7 \mathrm{a}=2.3 \pm 0.6$; Let $7 \mathrm{c}=2.2 \pm 0.2$; Let $7 \mathrm{~g}=2.8 \pm$ 0.5) (Figure 3). The largest increase for each miRNA studies occurred between 7 and 14 days after injury which preceded the development of hyperalgesia. 


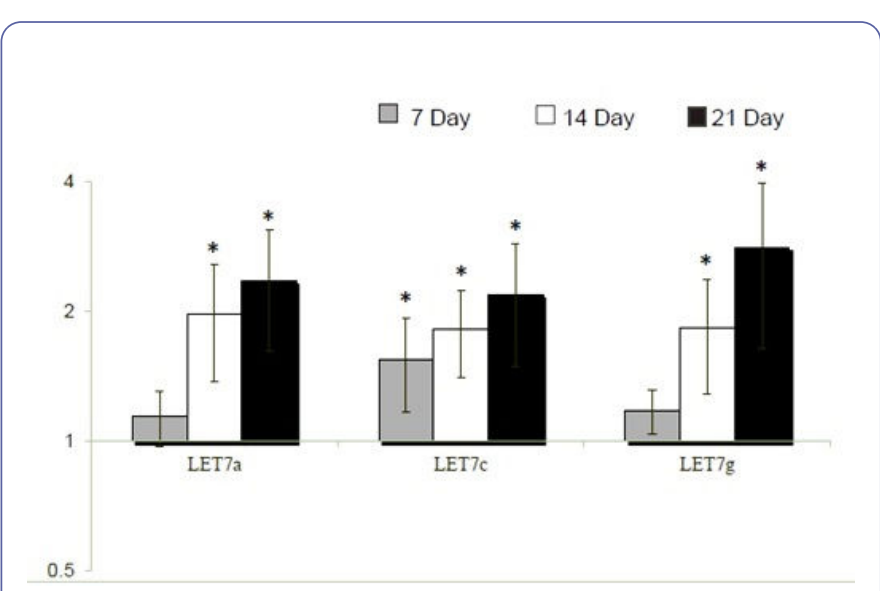

Figure 3 Fold changes of the 3 Let- 7 family members of interest in QUIS-injected animals ( $\mathrm{SCl}$ ) over time. Fold changes are relative to normalized miRNA levels in measured in SHAM animals. Let-7c was increased in QUISinjected animals compared to SHAM animals at $7(p=0.03)$ $14(p=0.01)$ and $21(p<0.01)$ day time points. Let-7a and Let-7g were increased in QUIS vs. SHAM animals at 14 and 21 days post-surgery $(p<0.01)$. All miRNAs studied showed a non-statistically significant trend toward increasing over time with levels being highest at the time corresponding to the presence of hyperalgesia after injury ( $p>0.05$ for all comparisons).

\section{Discussion}

Consistent with earlier studies [24], our data demonstrates that excitotoxic $\mathrm{SCl}$ induces hyperalgesia in rodent models and causes neuronal loss in the dorsal horn region. The novel finding is that all members of the Let-7 family of miRNAs studied were increased as much as 3 -fold in a time course parallel to the emergence of pain-related behaviors developed after $\mathrm{SCl}$. While several studies have analyzed miRNA expression and functional changes with $\mathrm{SCl}$, many are microarray studies providing conflicting information regarding overall patterns of these changes [25-27]. It has been suggested that many of these discrepancies may be attributable to model and severity of injury or even statistical analyses employed [19]. The Let-7 family of miRNAs has been shown to be up-regulated in response to $\mathrm{SCl}$ and has been linked to disruption in homeostatic cellular processes, such as apoptosis and cellular proliferation [21,28]. Through mRNA degradation and translational repression associated with these miRNAs, there is potential for their up-regulation to cause functional deficits in pain related structures [28]. One known effect of increased levels of the Let-7 family is repression of translation of the mu-opioid receptor, resulting in the loss of analgesic effect of opioids [20]. In animal models, SCI has been shown to reduce the expression levels of the mu opioid receptor (MOR) in the spinal cord $[12,29]$. The current data suggests that one mechanism behind the reduced number of MORs in the spinal cord after injury may be linked to upregulation of the Let-7 family of miRNAs. This reduction in MOR expression is likely to have significant impacts on the development of chronic pain as well as the diminished response to opioidergic drugs [30]. Importantly, in humans with $\mathrm{SCl}$, it has been shown that opioids are not universally effective in providing relief from $\mathrm{SCl}$-related pain syndromes $[10,31,32]$, suggesting that there may be an underlying change in the availability of functional state of the opioid receptors.

While we are able to recognize temporal associations between Let-7 miRNA levels and the development of pain after $\mathrm{SCl}$, current evidence does not allow us to directly link these two events. The degree of miRNA change needed to induce a behavioral response is unknown. Future studies should employee inhibitors of these specific miRNAs to determine if the behavioral outcomes, development of pain and response to opioids, can be modified after injury. This will help elucidate if changes in the Let-7 miRNAs are part of a general response to injury, or have functional implications that manifest as secondary complications of $\mathrm{SCl}$.

\section{Acknowledgements}

The authors would like to express their appreciation to Brian Whitfield, and Maurice Smith for technical assistance and Dr. Xiaoping Pan for guidance on RT-PCR experiments and manuscript preparation.

\section{References}

1. Baastrup C, Finnerup NB (2008) Pharmacological management of neuropathic pain following spinal cord injury. CNS Drugs 22: 455-75.

2. Attal N (2009) Chronic neuropathic pain management in spinal cord injury patients. What is the efficacy of pharmacological treatments with a general mode of administration? (oral, transdermal, intravenous). Ann Phys Rehabil Med 52: 124-141.

3. Cardenas DD, Felix ER (2009) Pain after spinal cord injury: a review of classification, treatment approaches, and treatment assessment PM R 1: 1077-1090.

4. Finnerup NB, Jensen TS (2004) Spinal cord injury pain-mechanisms and treatment. Eur J Neurol 11: 73-82.

5. Felix ER, Cruz-Almeida Y, Widerstrom-Noga EG (2007) Chronic pain after spinal cord injury: what characteristics make some pains more disturbing than others? J Rehabil Res Dev 44: 703-715.

6. Cruz-Almeida Y, Martinez-Arizala A, Widerstrom-Noga EG (2005) Chronicity of pain associated with spinal cord injury: A longitudinal analysis. J Rehabil Res Dev 42: 585-594.

7. Von Korff M (2005) Chronic spinal pain and physical-mental comorbidity in the United States: results from the national comorbidity survey replication. Pain 113: 331-339.

8. Widerstrom-Noga EG (1999) Perceived difficulty in dealing with consequences of spinal cord injury. Arch Phys Med Rehabil 80: 580-586.

9. Turner JA (2002) Catastrophizing is associated with pain intensity, psychological distress, and pain-related disability among individuals with chronic pain after spinal cord injury. Pain 98: 127-34. 
10. Teasell RW (2010) A systematic review of pharmacologic treatments of pain after spinal cord injury. Arch Phys Med Rehabil 91: 816-831.

11. Woller SA (2012) Analgesia or addiction?: implications for morphine use after spinal cord injury. J Neurotrauma 29 1650-1662.

12. Michael FM (2015) Contusive spinal cord injury up regulates muopioid receptor (mor) gene expression in the brain and down regulates its expression in the spinal cord: possible implications in spinal cord injury research. Neurol Res 37: 788-796.

13. Bhalala OG, Srikanth M, Kessler JA (2013) The emerging roles of microRNAs in CNS injuries. Nat Rev Neurol 9: 328-339.

14. Madathil SK (2011) MicroRNAs in CNS injury: potential roles and therapeutic implications. Bioessays 33: 21-26.

15. Liu NK (2009) Altered microRNA expression following traumatic spinal cord injury. Exp Neurol 219: 424-429.

16. Sakai A, Suzuki H (2015) microRNA and Pain. Adv Exp Med Biol 888: 17-39.

17. Jiangpan P (2016) Emerging Role of microRNA in Neuropathic Pain. Curr Drug Metab 17: 336-344.

18. Yunta M (2012) MicroRNA dysregulation in the spinal cord following traumatic injury. PLoS One 7: e34534.

19. Nieto-Diaz M (2014) MicroRNA dysregulation in spinal cord injury: causes, consequences and therapeutics. Front Cell Neurosci 8: 53.

20. He Y (2010) Regulation of opioid tolerance by let-7 family microRNA targeting the mu opioid receptor. J Neurosci 30: 10251-10258.

21. Liu G (2010) Cycling exercise affects the expression of apoptosisassociated microRNAs after spinal cord injury in rats. Exp Neurol 226: $200-206$
22. Hargreaves K (1988) A new and sensitive method for measuring thermal nociception in cutaneous hyperalgesia. Pain 32: 77-88.

23. Livak KJ, Schmittgen TD (2001) Analysis of relative gene expression data using real-time quantitative PCR and the 2 (Delta Delta C(T)) Method. Methods 25: 402-408.

24. Yezierski RP (1998) Excitotoxic spinal cord injury: behavioral and morphological characteristics of a central pain model. Pain 75 : 141-55.

25. Nakanishi K (2010) Responses of microRNAs 124a and 223 following spinal cord injury in mice. Spinal Cord 48: 192-196.

26. Izumi B (2011) MicroRNA-223 expression in neutrophils in the early phase of secondary damage after spinal cord injury. Neurosci Lett 492: 114-148.

27. Strickland ER (2011) MicroRNA dysregulation following spinal cord contusion: implications for neural plasticity and repair. Neuroscience 186: 146-160.

28. Liu NK, Xu XM (2011) MicroRNA in central nervous system trauma and degenerative disorders. Physiol Genomics 43: 571-80.

29. Obara I (2009) Local peripheral opioid effects and expression of opioid genes in the spinal cord and dorsal root ganglia in neuropathic and inflammatory pain. Pain 141: 283-291.

30. Kohno T (2005) Peripheral axonal injury results in reduced mu opioid receptor pre- and post-synaptic action in the spinal cord. Pain 117: 77-87.

31. Smeal WL (2006) Spinal cord injury. J Pain 7: 871-877.

32. Attal N (2002) Effects of IV morphine in central pain: a randomized placebo-controlled study. Neurology 58: 554-563. 